\title{
EL PROFESOR UNIVERSITARIO VENEZOLANO MIGRANTE: ¿EN BÚSQUEDA DE LA PRODUCTIVIDAD PERDIDA?
}

\author{
VENEZUELAN MIGRANT PROFESSORS: \\ IN SEARCH OF THEIR LOST PRODUCTIVITY?
}

Ramón Alexander Uzcátegui Pacheco*, Audy Salcedo**

\section{Resumen}

Ante el fenómeno creciente de la migración venezolana, nos interesó conocer las razones por las cuales emigra el profesorado universitario venezolano. Una migración masiva como la que experimenta el país caribeño incluye el éxodo de talento humano, de personal calificado. Metodológicamente, la investigación es descriptiva. Se identificó en una muestra de profesores universitarios las razones por las cuales decidieron emigrar. Se empleó la técnica del muestreo por bola de nieve a partir de la distribución de una encuesta en la que participaron más de 350 profesores distribuidos en distintos países. Entre los resultados se destaca que los profesores emigran en búsqueda de un mejor futuro; optan por nuevas y mejores condiciones de vida. Desde la perspectiva académica, la migración es una oportunidad para recuperar la productividad perdida a causa del desmejoramiento en las condiciones de vida personales y profesionales de los académicos venezolanos. Los profesores emigran por varias razones, pero en la base está la idea, el proyecto personal de continuar con sus vidas académicas y agregar valor a sus capacidades en otras latitudes.

* Profesor en el área de Formación General, Universidad Andrés Bello, Chile. email: r.uzcateguipacheco@uandresbello.edu, ORCID https://orcid.org/0000-0002-5669-6663

** Profesor de Estadística e Informática Aplicada a la Educación, Universidad Central de Venezuela, Venezuela. email: audy.salcedo@ucv.ve, ORCID https://orcid.org/0000-00029783-8509 


\title{
Palabras claves:
}

Migración, migración laboral, personal académico docente, universidad, productividad laboral, Venezuela.

\begin{abstract}
Given the growing phenomenon of Venezuela's emigration, we were interested in knowing the reasons why Venezuelan professors emigrate. Massive emigration like the one this Caribbean country is experiencing includes the exodus of valuable human talent, of very qualified personnel. Methodologically, this research is descriptive. The reasons why they decided to emigrate were identified in a sample of migrant professors. A technique of snowball sampling was used, based on the distribution of a survey in which more than 350 professors located in various countries participated. Among the main results, it is worth noting that professors emigrate in search of a better future; they look for new and better living conditions. From the academic perspective, emigration represents an opportunity to restore lost productivity due to the deterioration in both personal and professional living conditions of Venezuela's academics.

Professors migrate for various reasons but the main one seems to be their personal project of going on with their academic lives and enhancing their capacities in other countries.
\end{abstract}

\section{Keywords:}

Migration, highly skilled migration, professors, university, academic productivity, Venezuela.

\section{Introducción}

Este artículo expone los resultados de una investigación acerca de la reciente migración venezolana que afecta específicamente al profesorado universitario. Ante el fenómeno creciente de la migración venezolana, nos interesó conocer las razones por las cuales emigra el profesorado universitario de este país. Las causas de la migración venezolana han sido caracterizadas en diferentes medios 
y espacios, tanto académicos e institucionales, como por organizaciones no gubernamentales. Sin embargo, interesa conocer cómo y por qué este segmento de profesionales venezolanos tomó la decisión de abandonar su país, considerando su formación profesional y su estatus laboral en la sociedad venezolana.

La información ofrecida por la Organización de las Naciones Unidas (ONU); la Organización de las Naciones Unidas para la Educación, la Ciencia y la Cultura (UNESCO); el Fondo de las Naciones Unidas para la Infancia (UNICEF) y la Organización Internacional para las Migraciones (OIM), indican que Venezuela vive un proceso de migración masiva. Según la Oficina del Alto Comisionado de las Naciones Unidas para los Refugiados [ACNUR] más de 4,7 millones de ciudadanos han salido del país en los últimos años, en busca de cobijo en otras partes del mundo. El 49\% de las familias venezolanas afirma que al menos uno de sus miembros ha emigrado del país y para julio de 2019 se estimaba que entre el 15\% y el 19\% de los venezolanos había emigrado. Esto significa que entre 4.771.216 y 6.047.340 de personas decidieron buscar nuevas opciones fuera de Venezuela (Consultores 21, 2019).

La migración masiva usualmente ocurre en situaciones de catástrofe natural o sociocultural; sin embargo, la salida de ciudadanos venezolanos no se debe a una catástrofe natural o a una guerra, sino a la situación de crisis institucional, política, social y económica que vive el país desde 1999. La muestra de los migrantes puede ser tan representativa como el universo de ciudadanos que integran el país. Una migración de estas características considera por igual a ciudadanos pobres, clase media, clase alta; propietarios y no propietarios, escolarizados y no escolarizados, niños, jóvenes y adultos, mujeres y hombres, empleados, dependientes y emprendedores, personal calificado y no calificado; alumnos, profesores, directivos, funcionarios de política y gestión pública, entre otros.

En esta investigación, estudiamos un segmento de esa amplia migración venezolana. Se trata de una parte de la migración calificada: los profesores universitarios. Al respecto, la universidad venezolana atraviesa circunstancias muy adversas, las que han limitado su funcionamiento y el cumplimiento de las funciones del 
profesorado (docencia, investigación, extensión y gestión). A pesar de ello, muchos profesores se mantuvieron (y se mantienen) en sus actividades, pero para una parte de estos docentes las circunstancias se hicieron extremas, por lo cual vieron en el extranjero la posibilidad de emprender una nueva vida y, en el mejor de los casos, dar continuidad a sus expectativas de desarrollo académico y de vida profesional.

La presente investigación es de carácter descriptivo. A partir de una muestra de profesores universitarios se identifican algunas razones por las cuales estos profesionales decidieron salir del país. En el análisis se consideraron factores como: el perfil profesional del profesor, el área de conocimientos en la que se formó y se desempeñaba, así como su productividad académica antes de salir de Venezuela. El análisis de la información recabada permite concluir que el profesorado universitario venezolano emigra con la aspiración de retomar sus actividades universitarias (docencia, investigación y extensión) y para darle continuidad más allá de las fronteras de su país.

\section{Marco Teórico}

Según datos de la Oficina Internacional de Migraciones, casi 5 millones de venezolanos habían salido de su país en los últimos años. El mayor flujo migratorio correspondía al año 2014 en adelante. Esta situación conduce necesariamente a precisar marcos referenciales por los cuales el fenómeno migratorio venezolano pueda ser estudiado más allá de las premisas conceptuales del movimiento de población en el mundo (Páez y Phelan, 2019) y desde la complejidad del fenómeno, considerando la multidimensionalidad y dinamismo de la movilidad humana (OIM, 2012). La migración venezolana puede ser explicada según variables endógenas, propias de la dinámica socioeconómica y política del país. En los últimos años la migración ha crecido en forma exponencial (García, et al., 2019), transformándose en una dimensión de la crisis que experimenta el país, "no es un tema exclusivo de las clases altas o medias, sino que 
hay una tendencia en crecimiento de la emigración desde la pirámide social, es decir, personas de los estratos más bajos de la población” (Vargas, 2018, p. 117).

Mesa y Phelan (2018) señalan que el migrante venezolano tiene unas características peculiares al provenir de una economía petrolera rentista y una cultura democrática aprendida entre 1958 y 1999: "el venezolano que emigra por lo general tiene niveles importantes en cuanto a logros personales (formación, vivienda, movilidad, ahorros, trabajo y familia) pero tiene limitaciones en la posibilidad de escoger y dar valor a las capacidades adquiridas" (p. 40).

Una migración masiva como la venezolana, involucra el éxodo de talento humano y de personal calificado. Entre 1990 y 2007 el inventario de migrantes calificados venezolanos en países de la Organización para la Cooperación y Desarrollo Económicos (OCDE) registró una variación del 216\% (Freitez, 2019). La Coordinadora Regional de Investigaciones Económicas y Sociales (CRIES) y la Stanley Foundation (2017) señalan que en un inicio la migración venezolana estuvo constituida por profesionales de clase media y clase media alta, con edades entre los 25 y 40 años, lo que significaba una pérdida importante de su población económicamente activa, así como de capacidades intelectuales.

Pero ya no solo emigra una élite representada en sus cualidades profesionales, también se movilizan otros ciudadanos que ven alteradas sus condiciones de vida y posibilidades de desarrollo: "La emigración reciente ha transversalizado todo el espectro social, pero todavía tiene relevancia la población con educación universitaria y que pertenece a estratos sociales más aventajados” (Freitez, 2019, p. 10). La transversalización de la migración, en todo el espectro social venezolano, tiene dos razones comunes entre las muchas que pueden influir en la vida de las personas: razones políticas derivadas de las contradicciones que supone el establecimiento de un proyecto político de carácter autoritario, donde las instituciones del Estado no atienden las necesidades del ciudadano común y, por otro lado, razones económicas por la crisis que hace mella en las posibilidades efectivas de bienestar de la población y la imposibilidad de acceder a mejores condiciones de vida por la vía del trabajo digno. 
De estos factores se deriva una lista de condicionantes en cuanto a inseguridad, desabastecimiento, hiperinflación, entre otros y, como se registra en 2020, carencia de servicios básicos como agua, luz, internet, medios de transporte, gasolina, gas natural, entre otros.

La migración calificada, que históricamente ha sido selectiva, hoy día en el caso venezolano es masiva. Los profesionales se desplazan más allá de las fronteras nacionales, no atraídos por receptivos mercados de recursos humanos, sino por la dinámica interna el país de origen. A pesar de que el destino es muchas veces incierto, emigran con ideas y proyectos claros como mejorar su vida productiva y profesional para su propio bienestar, el de sus familiares y de la sociedad, en general. Estos profesionales ven fuera de Venezuela la posibilidad de continuar con su proyecto de vida en un lugar que les ofrezca mejores condiciones para hacerlo, aun cuando esto signifique la pérdida del ambiente en el cual se había iniciado, desarrollado y alcanzado una posición de relevancia dentro de su contexto profesional.

En el caso de los profesores, acceder a la docencia universitaria exige del profesional cualidades específicas en el dominio del conocimiento de su área del saber para tener la posibilidad de enseñar en esta área (Parra-Sandoval, 2003). Además, exige la adquisición o fortalecimiento de otras competencias como la investigación, la extensión y la gestión universitaria. Ser parte del profesorado universitario exige condiciones favorables para el desempeño. Actualmente, tal como sostienen Linarez y Linarez (2019), los docentes universitarios venezolanos no disponen de recursos para la movilidad social, ni para asistir a encuentros científicos internacionales. Incluso sus condiciones de salud no están atendidas, al no poseer un sistema que les brinde cobertura.

La dependencia fiscal de las universidades, con respecto al Estado, ha sido un factor fundamental en la dependencia de estas instituciones educativas. La reconducción y congelamiento del presupuesto universitario por parte del Ejecutivo Nacional ha limitado el funcionamiento de las altas casas de estudios (Hora Universitaria, 2013). Pese al esfuerzo institucional de las universidades, particularmente las autónomas, de avanzar, a pesar de las políticas guber- 
namentales, la precarización del trabajo académico universitario ha sido uno de los signos de la época desde el advenimiento de la Revolución Bolivariana (Ramírez, 2020).

Desde el año 2000, y particularmente a partir de 2005, se intensificó el control hacia las universidades autónomas y las pocas experimentales. El estancamiento de las universidades incluye una política de disminución presupuestaria, con aporte de recursos casi exclusivamente para sueldos de los trabajadores. A eso se le agrega el uso del Poder Judicial para vulnerar el ejercicio de la autonomía universitaria, lo cual produjo, por ejemplo, la suspensión ilegítima de los procesos electorales de las distintas universidades, así como la interrupción de los concursos de oposición de los profesores (Aula Abierta, 2018).

Desde 2007, el gobierno de Venezuela mantiene a las universidades con el mismo presupuesto. Todos los años, cada universidad envía al Ejecutivo Nacional un presupuesto sustentado en sus distintas necesidades, pero el gobierno rechaza los presupuestos y asigna la misma cantidad que en el año 2007, en lo que se denomina los presupuestos reconducidos. Para cubrir las diferencias de cada año, las universidades dependen de los llamados créditos adicionales, esto es, la asignación de forma unilateral por parte del gobierno, de montos extras. Estos créditos adicionales se asignan varias veces en el año, sin ninguna planificación. Esta situación no se corresponde con la dinámica institucional de las universidades, ni con la realidad macroeconómica del país, afectado por una inflación crónica de más de tres dígitos al año e hiperinflación desde 2017. En 2020 señala Benjamín Scharifker, rector de la Universidad Metropolitana que "la deserción estudiantil y profesoral ronda en promedio 40\%" (Descifrado, 2020), lo que representa un verdadero éxodo de talentos en el sector.

A la par, se implementa una política restrictiva que disminuye los salarios de los profesores, lo cual tiene consecuencias negativas en la subsistencia de los docentes y la de sus familias (Torres, et al., 2020). Otros componentes de la situación universitaria son: falta de recursos para el mantenimiento de la planta física, bajas asignaciones presupuestarias a la investigación, constantes agresiones a los 
universitarios por parte de grupos parapoliciales y acoso del hampa común, sin que el Estado aplique correcciones (Bravo, 2014; 2018; Singer y Moleiro, 2020).

En Venezuela, la carrera académica, acceder a ella y cumplir su normalmente largo ciclo formó parte del fortalecimiento de la institucionalidad educativa y del desarrollo y diversificación del ecosistema de ciencia y tecnología. También, de las posibilidades que ofrecía la sociedad en términos de movilidad y ascenso social (Parra-Sandoval, 2003; Morles, et al., 2003). El acceso a la escolaridad y en particular a la escolaridad universitaria significó para muchos la modernización y mejoramiento no solo de sus vidas, sino de la sociedad en general.

Pero la profesión de profesor universitario se ha visto sustantivamente alterada por la dinámica interna de la política, la economía y el funcionamiento institucional. Por ejemplo, la política salarial unilateral aplicada por el gobierno iniciado en 1999 ha llevado a los profesores a una pérdida en sus ingresos. Los salarios oscilan entre una proporción del $-1,710.26 \%$ y $-2,806.73 \%$ con respecto a los salarios devengados en el año 2001 (Torres et al., 2020). Hoy rondan en los 4 y 5 dólares estadounidenses mensuales para un profesor con la más alta calificación del escalafón universitario. El deterioro es tal que un profesor de mayor escalafón y dedicación, en consecuencia, el de mayor salario en la universidad venezolana, no logra cubrir ni siquiera el 10\% del costo de la canasta alimentaria nacional (Tovar, 2020).

Para muchos profesionales, el tiempo dedicado a la sobrevivencia es superior a todo lo demás. Esto sobre-exige sus capacidades efectivas para mantener los indicadores y estándares de productividad deseados en sus campos profesionales. Es por esta, entre otras razones, que el profesorado puede considerar la migración como alternativa. Sin duda que podrá marcharse con la idea de mejorar sus condiciones de vida, pero seguramente no perderá de vista la posibilidad de seguir desarrollando su carrera académica, aunque ello signifique empezar de nuevo, en el mejor de los casos, en los países de acogida. Pero, además, para un profesor universitario, para un académico, "la movilidad se considera un valor intrínseco a la carrera científica” (Masanet, et al., 2020, p. 51). La movilidad 
se presenta como una posibilidad de ampliar el repertorio de experiencias, contactos y ámbitos en el que su hacer cobre relevancia particular. En este sentido, "la movilidad permite oportunidades laborales por el establecimiento temporal de una proximidad física entre científicos/as que refuerza otras proximidades (social, cognitiva, organizacional)" (Masanet, et al., 2020, p. 51). La experiencia migratoria, con una política de retorno y de transferencia de capital intelectual, puede traducirse en oportunidades de redes de intercambio entre los académicos de los países receptores y los de países de origen de los migrantes.

Así, la evidencia nos indica que el compromiso con la formación de talentos en sus respectivas áreas, y la generación de conocimiento en sus campos de saber, es una meta importante del profesor universitario migrante. Tal vez la movilidad del profesorado universitario se pueda explicar por la necesidad de seguir salvaguardando las posibilidades de hacer ciencia en un terreno menos árido del que se procede. Pero a pesar de su menor vulnerabilidad respecto al resto de los migrantes, actualmente la integración de estos profesionales calificados se ve afectada por las consecuencias de las crisis económicas (Pellegrino, 2013, p. 10).

\section{Metodología}

Metodológicamente, se trata de una investigación descriptiva (Hernández et. al, 2006). Se emplearon parámetros cuantitativos y cualitativos en el tratamiento de los datos, por lo que puede considerarse investigación mixta, en la que se busca identificar, a partir de una muestra de profesores universitarios, las razones por las cuales decidieron emigrar de Venezuela. Partimos de la hipótesis que los profesores universitarios emigran en busca de mejores condiciones sociales que les permitan continuar con su proyecto de vida académica, ideas que contrastan con estudios sobre migración calificada, según los cuales este movimiento de población se explica por la dinámica del mercado laboral global (Cohen, 2001; Domeniconi y Jarochinski, 2018; OIM, 2019; UNESCO, 2019). En este caso, 
la explicación parte de las variables internas del país (De la Vega y Vargas, 2014; Páez, 2010; Zúñiga, 2011; Requena y Caputo, 2016; Vargas, 2018), donde la posibilidad de migrar está asociada a la idea de continuar con el proyecto de vida académica que los profesores universitarios, considerados como migración calificada, tenían al momento de salir de su país de origen.

En esta investigación, se consideraron las siguientes variables: identificación del profesor, aspectos afectivos-familiares, situación laboral antes y después de salir de su país; situación en su carrera académica, decisión u opción por migrar, proceso migratorio y vínculos con Venezuela. En este artículo se reportan resultados de algunas de las dimensiones consideradas en la investigación tales como características generales, actividades académicas y productividad académica en el país de acogida.

La recolección de los datos se realizó por medio de un cuestionario en línea, diseñado ad hoc y distribuido por correo electrónico. En el instrumento, se incorporaron preguntas de selección múltiple y preguntas abiertas para que los profesores complementaran con su opinión acerca de los asuntos abordados. El instrumento diseñado consta de 56 preguntas. El cuestionario fue validado mediante juicio de expertos por 10 investigadores, 5 del área de educación y migración y 5 de metodología. La opinión de los 10 validadores permitió introducir modificaciones en el cuestionario. Este instrumento estuvo disponible durante tres meses, tiempo en el cual se obtuvieron 373 respuestas.

Previamente se consultó a los sujetos si estarían dispuestos a formar parte de una investigación sobre profesores migrantes venezolanos en calidad de informantes clave. Se les notificó que los datos personales de los participantes tendrían carácter confidencial y que las preguntas del instrumento de investigación estaban dirigidas a conocer acerca de su situación migratoria y su productividad académica antes y después de salir de Venezuela.

En el proceso de investigación se utilizó el muestreo por bola de nieve, técnica no probabilística utilizada con frecuencia para acceder a poblaciones de baja incidencia y a individuos de difícil acceso por parte del investigador (Hernández et al., 2006). Tal es el caso de 
los profesores migrantes, en que los investigadores desconocían su real ubicación. Luego de identificar a los primeros profesores que habían migrado, se les pidió ayuda para ubicar a otras personas que tuvieran un perfil similar. El procedimiento se repitió hasta conformar el grupo de elementos o cuestionarios que se analizarían.

Las preguntas de selección múltiple se trataron cuantitativamente a partir de parámetros estadísticos descriptivos, frecuencias y porcentajes, lo que permitió caracterizar a la población objeto de estudio. Las preguntas abiertas se trataron de forma cualitativa a partir de la técnica de análisis de contenido de los planteamientos expresados por los profesores. Las preguntas abiertas ofrecen la posibilidad a los encuestados de responder, expresando sus opiniones o razonamientos en torno a las interrogantes planteadas

En la presentación de los resultados, los análisis cuantitativos de los datos fueron acompañados con los análisis de las ideas expresadas por los sujetos de la investigación. Se incorporaron frases textuales de los consultados identificados como SujetoN (S1...). Esto respetando el anonimato de los encuestados y para darle profundidad a las estadísticas de la investigación. Se busca así mostrar las opiniones expresadas por los profesores y ayudar a comprender las dimensiones del problema y el impacto que éste tiene en los sujetos objeto de esta investigación. Este abordaje se aviene con orientaciones teóricas y metodológicas en las actuales tendencias de la investigación sobre migración, donde las opiniones de los migrantes juegan un papel esencial en la comprensión de las dinámicas específicas de las personas y grupos humanos. Se trata de una lectura que complementa las cifras sobre la migración venezolana y la migración en general.

\section{Resultados}

\subsection{Características generales}

La muestra de 373 docentes proviene de 14 universidades venezolanas, la mayoría de ellas del sector público (89\%). Aunque la 
ubicación geográfica de las universidades cubre casi todo el país, hay un sesgo importante de docentes de una Universidad pública, situada en Caracas, Venezuela (42,1\%), la más antigua del país. El $74,5 \%$ del grupo señaló que eran profesores a tiempo completo o a dedicación exclusiva en su institución para el momento de emigrar. Esto significa que dedicaban por lo menos 36 horas semanales a la universidad, lo cual se traduce en una pérdida cuantiosa de horas de clase y de investigación para la universidad venezolana.

El 50\% de los encuestados es de sexo femenino y $71 \%$ indican estar casados o con pareja. Son en su mayoría venezolanos por nacimiento y solo $24 \%$ manifestó tener una segunda nacionalidad. La mayoría tienen edades entre los 36 y 65 años, siendo los profesores entre 36 y 45 años el número mayor de participantes, lo que representa un $28 \%$ de la muestra. Luego hay un $25 \%$ con edades entre $46 y$ 55 , mientras que $22 \%$ tienen edades entre 56 y 65 años. Es un grupo de docentes en edad activa, en plena capacidad para el desarrollo de su carrera académica.

Los consultados señalaron que eran personal fijo en sus instituciones, profesores ordinarios en el escalafón universitario, quienes ya habían iniciado su carrera académica. Apenas 68 profesores de los 373 consultados indicaron que estaban en la condición de contratados para el momento de emigrar. El 31\% señaló que tenía grado de Magister y $47 \%$ que tenía Doctorado, con lo cual se ratifica que se trata de una migración de alta calificación. A los encuestados se les consultó sobre los años de su vida profesional dedicados a las labores universitarias. Con esa información se elaboró la tabla 1.

En la tabla 1 se observa que $49 \%$ de los profesores migrantes tenían 12 años o menos, trabajando en la universidad. Este es el grupo que podría considerarse la generación de relevo, los llamados a llevar adelante la universidad en los próximos 10 ó 15 años en distintas áreas, pero principalmente en docencia e investigación. Otro grupo para destacar es el que tiene entre 17 y 24 años de servicio, quienes representan el 16,9\% de los encuestados. Todos ellos estaban relativamente cerca de haber cumplido el tiempo de servicio necesario para la jubilación (25 años). 
Tabla 1. Años de servicio en la universidad antes de emigrar

\begin{tabular}{ccc}
\hline Rangos & N & Porcentaje \\
\hline $1-4$ & 65 & 17,4 \\
$5-8$ & 66 & 17,7 \\
$9-12$ & 53 & 14,2 \\
$13-16$ & 31 & 8,3 \\
$17-20$ & 43 & 11,5 \\
$21-24$ & 20 & 5,4 \\
$25-28$ & 45 & 12,1 \\
$29-32$ & 17 & 4,6 \\
$33-36$ & 14 & 3,8 \\
37 o más & 19 & 5,1 \\
\hline Total & 373 & \\
\hline
\end{tabular}

Fuente: Elaboración propia

En la tabla 1 también se observa que el $25,5 \%$ de docentes al momento de su salida del país tenía 25 años o más de servicio, tiempo suficiente para jubilarse. Con frecuencia el profesorado universitario venezolano sobrepasaba el tiempo requerido para la jubilación $\mathrm{y}$, continuaba trabajando para mantener labores de investigación en la institución.

Al ser consultados los profesores sobre la relación laboral con su institución, las principales respuestas fueron que: había renunciado 200 (53,6\%), que estaba jubilado 106 (28,4\%), y 52 (13,9\%) que tenían algún tipo de permiso. Esos dos primeros grupos ya no tienen vinculación laboral alguna en Venezuela y podrían optar por quedarse en el país de acogida. El tercer grupo (con permisos) podría ser de aquellos que van a explorar el terreno en el país receptor para luego tomar la decisión de quedarse o no. Pese a que la investigación no hace seguimiento de este grupo, se identifican casos en que la decisión final fue la renuncia a la universidad y emprender en los países receptores. 
A la pregunta de, ¿Teniendo posibilidades de desarrollar su carrera académica en Venezuela, por qué emigraron?: El 63\% de los encuestados señala como primera razón para emigrar la situación política del país, mientras que como segunda razón señalan la situación económica (29\%). Al solicitarles especificar los motivos de su migración, los encuestados señalan como principales: Búsqueda de un mejor futuro (71\%) y optar por nuevas condiciones de vida $(15,5 \%)$. Lo anterior ratifica lo señalado por otros investigadores (Lares, 2014; Sánchez y Massey, 2014), en cuanto a que la migración de venezolanos altamente calificados está vinculada con aspectos estructurales de la Venezuela actual.

\subsection{Actividades académicas}

En su vida laboral, el profesor universitario asume varias actividades como parte de su compromiso con la universidad. Las más comunes son docencia, investigación y extensión. Luego, en función de sus actitudes y aptitudes, podrá asumir actividades de liderazgo y gestión en la estructura universitaria. En esta investigación, consultamos sobre aspectos relacionados con la docencia, la investigación y la gestión universitaria. A continuación, describiremos las actividades que desarrollaban los docentes antes de emigrar de Venezuela.

\subsubsection{Docencia}

¿Cómo es dar clases en tiempos de la Revolución Bolivariana? A los profesores universitarios migrantes se les consultó sobre las condiciones para ejercer la docencia en su universidad hasta el momento de su salida. Los resultados se muestran en el gráfico 1.

Solamente el 6,1\% de los docentes consultados considera que sus condiciones para ejercer la docencia eran buenas o muy buenas para el momento de su egreso. En contraste, el 73,2\% señala que sus condiciones eran malas o muy malas. Cuando se habla de condiciones para ejercer la docencia de inmediato se puede pensar en variables relacionadas con los recursos que proporciona la institución para dar las clases, pero también hay que incluir otras 
variables como salarios, posibilidades de traslado hacia la universidad, escasez de dinero en efectivo, acceso a alimentos y seguridad ciudadana, entre otras.

Gráfico 1. Condiciones para ejercer la docencia en su universidad

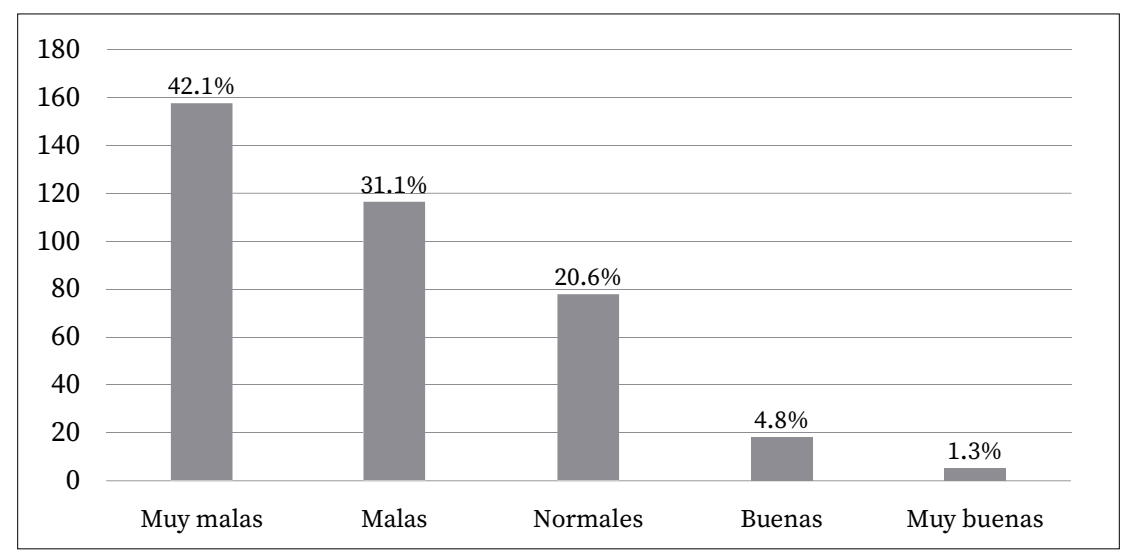

Fuente: Elaboración propia

Hay variables que probablemente en otros países no se considerarían, pero en el caso de Venezuela son fundamentales: el acceso a los servicios básicos en la universidad. Es frecuente que las universidades venezolanas tengan problemas de suministro de agua, luz eléctrica, gas natural, acceso a internet y seguridad. Son frecuentes los boletines de prensa de las universidades y las denuncias en las redes sociales, señalando la ausencia de uno o más servicios, por ejemplo: "La sede de Sartenejas de la Universidad Simón Bolívar (USB) cumple hoy dos semanas sin recibir suministro de agua potable por parte de Hidrocapital" (USB, 2020, p. 1). Pero además la delincuencia también afecta a las universidades, ya sea de forma individual (asaltos a miembros de la comunidad) o institucional (robo de equipos). "Encapuchados asaltaron a estudiantes de la Universidad de Oriente (...) los malhechores ingresaron de manera violenta y quitaron a un aproximado de 30 personas sus pertenencias como celulares, calculadoras, artículos escolares y prendas” (Rondón, 2017, p. 1). 
Hay centros universitarios que sufren de robos reiteradamente, tal es el caso del Instituto de Medicina Tropical de la Universidad Central de Venezuela, que ha sufrido 76 robos (Castro, 2020). Son situaciones que afectan por igual a docentes, estudiantes, personal obrero, administrativo y de servicio, trastocando el normal funcionamiento de la institución y modificando las condiciones para la docencia.

El conjunto de tales variables lo reportan los encuestados cuando se le solicitan detalles sobre las condiciones en las cuales ejercían la docencia. En las respuestas abiertas, con relación a lo que consideran muy malas, los sujetos (S) consultados señalan: "Los estudiantes no tenían cabeza para estudiar, lo único que se hablaba era de las marchas" (S8); "Era bastante difícil adquirir los elementos básicos para impartir clases o hacer vida académica dentro de la universidad (elementos de papelería, de higiene, etc.)" (S42); "Sin recursos para trabajar dignamente" (S85); "Sueldos de miseria, condiciones mínimas para trabajar, instalaciones deterioradas, falta de presupuesto para investigar, falta de apoyo para asistir a eventos, para publicar y para formación" (S103). Las malas condiciones se expresan en el siguiente sentir: "Me fui en 2015 y las condiciones de la universidad eran ya muy precarias" (S04); "Cortes eléctricos, no disponibilidad de internet, inseguridad en los salones" (S44); "Hace 8 años se podía trabajar. Salí para volver, pero no pude. Desde ese momento hasta hoy todo ha empeorado. No se gana lo suficiente para mantener una familia” (S106).

Con relación a lo que se considera "normal” y "buenas" destacan: "En la universidad todavía había muy buenas condiciones para la docencia y la investigación, pero ya el país se estaba viniendo abajo" (S45); "Se podía asistir a la universidad, pero cada vez era más difícil, los equipos se estaban deteriorando rápidamente” (S70). La conjugación de factores externos e internos a la universidad condicionan la actividad del docente, pero de acuerdo con lo relatado por la mayoría de los consultados la situación no es la normal de cualquier país.

El profesorado migrante que integra la muestra desarrollaba actividades docentes en distintos campos del saber. La tabla 2 muestra las áreas de conocimiento de adscripción para el momento de su salida del país. 
Tabla 2. Área de conocimiento de adscripción en Venezuela

\begin{tabular}{|c|c|}
\hline Área de Conocimiento & $\mathbf{N}$ \\
\hline Ciencia y Tecnología (Arquitectura, Ciencias, Ingeniería) & 100 \\
\hline Ciencia de la Salud (Farmacia, Odontología, Medicina) & 37 \\
\hline $\begin{array}{l}\text { Ciencias Sociales (Ciencias Económicas y Sociales, Humanidades } \\
\text { y Educación, Ciencias Jurídicas y Políticas) }\end{array}$ & 181 \\
\hline Ciencias del Agro y del Mar (Agronomía, Ciencias Veterinaria) & 15 \\
\hline Total & 333 \\
\hline
\end{tabular}

Fuente: Elaboración propia

El área de conocimientos donde más se registran profesores migrantes incluye aquellas asociadas con las ciencias sociales. Dentro de esta área del saber los que más han emigrado son los profesores vinculados con las humanidades (historia, geografía, artes y filosofía) y la educación. En ciencia y tecnología los registros indican que los profesores que más han emigrado son los de ciencias básicas (química, física y matemáticas). Mientras que en ciencias de la salud, el componente que más ha emigrado, conforme a la muestra contactada, es el de medicina. Las áreas de ciencia y tecnología y ciencias de la salud suelen ser áreas prioritarias en la mayoría de los países, por lo que el posible ingreso de esos docentes a las universidades del extranjero sería más fácil.

La salida de este importante grupo de docentes podría resentir en diversas formas a las cátedras y departamentos a las que pertenecían, a saber: (a) limitación de la oferta académica, (b) sobrecarga de los profesores activos, (c) modificación de planes de estudio. Además, de provocar un retraso en la graduación de los estudiantes o profundizar la deserción de estos, al ver que no les es posible cursar de forma regular las asignaturas de su plan de estudios. 


\subsubsection{Investigación}

La investigación es una función esencial de la institución universitaria. En el caso de Venezuela el ecosistema de producción científica y tecnológica del país se encuentra casi exclusivamente en las universidades (MCT, 2005; Requena y Caputo, 2016; Según la Oficina del Alto Comisionado de las Naciones Unidas (Ramírez y Salcedo, 2016). De allí la importancia de consultar al grupo participante en torno al tema de la investigación y otros relacionados como la carrera académica y la producción de artículos.

El gráfico 2 resume las respuestas dadas por los encuestados sobre las condiciones de que disponían para hacer investigación antes de su salida del país.

Gráfico 2. Condiciones para hacer investigación en la Universidad

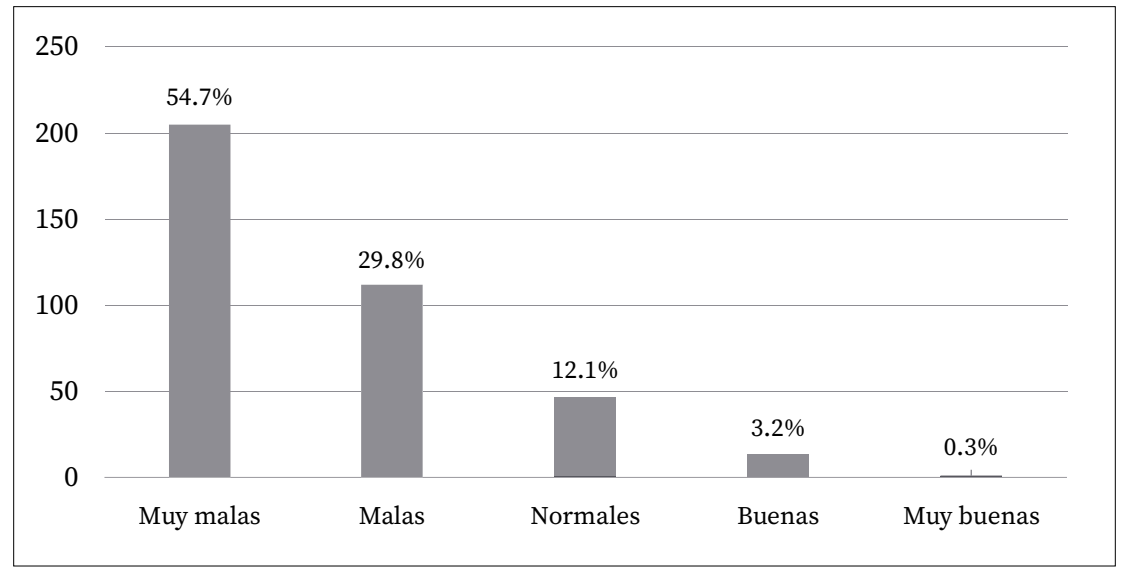

Fuente: Elaboración propia

Las opiniones del profesorado respecto a las condiciones para la investigación coinciden con lo expresado antes respecto de la docencia, por cuanto ambas funciones se realizan en la misma institución y poseen circunstancias comunes. No obstante, un aspecto que diferencia la investigación de la docencia es el acceso a financiamiento especial para la actividad, los investigadores deberían lograr ese 
financiamiento principalmente por vía del presupuesto universitario o con el gobierno mediante la Ley Orgánica de Ciencia, Tecnología e Innovación (LOCTI). Los presupuestos de las universidades son definidos de manera unilateral por el gobierno sin tomar en cuenta las solicitudes de las instituciones, en ellos más del 90\% de lo asignado se destina a pagar sueldos del personal universitario. Las asignaciones para investigación son simbólicas. Las opiniones de los investigadores dan cuanta de la insuficiencia presupuestaria para apoyar la investigación en las universidades. La LOCTI tiene previsto aportes de las empresas para financiar la investigación, no obstante, modificaciones hechas por el gobierno venezolano a esa ley en 2011, no permiten que el aporte de las empresas llegue directamente a las universidades (León, 2014; De la Vega y Vargas, 2014). La falta de financiamiento y otros factores hacen muy difícil llevar a cabo investigación en las universidades venezolanas.

Cuando se pide a los profesores detalles de las condiciones que tenían para hacer investigación en su universidad al momento de su salida, las respuestas son variadas. Entre las respuestas correspondientes a "muy malas" se señalan: "El financiamiento a proyectos de investigación son (sic.) irreales" (S4); "La situación de inflación, devaluación de la moneda con relación al presupuesto universitario reconducido de las universidades desde 2007, hacía que el reglón de investigación dentro del presupuesto fuera poco relevante. En la UPEL el monto asignado para la investigación era irrisorio" (S14); "No había fuentes de financiamiento ni apoyo del gobierno al control de gestión de las instituciones públicas. Enfrentamiento total entre gobierno y universidades" (S45); "La investigación, la poca que se hacía era autofinanciada, de carácter documental, en un contexto donde no hay actualización de bibliotecas, no había acceso a revistas, actualización de libros en la biblioteca, apoyos de asistencia a congresos (ni siquiera a nivel nacional)" (S37); "No había fondos para viajar o cubrir los costos de inscripción, nunca pude ir a un evento. Toda la información y apoyo debía ser subvencionada por cuenta propia" (S80).

Las consideraciones en cuanto a "malas" siguen en la misma línea argumentativa: "No había recursos para nada, no se tenía 
acceso a libros, no se contaba con tiempo, ni ayudantes, ni recursos materiales para poder hacer un estudio". (S05); "Las condiciones eran precarias, se carecía de todo" (S13).

Cuando se dice que las condiciones eran "buenas" refieren a la calidad de los investigadores, más que a las condiciones; (antes había) "Tiempo para dedicarle a la investigación, ya que la carga académica se limitaba a dos materias, excelentes servicios de la biblioteca central y del Decanato de I+D" (S85); "Las fuentes de financiamiento y los presupuestos no se sostienen en el tiempo. Ningún tipo de respaldo ni fomento a la investigación. Cuando me fui, había entregado el trabajo de ascenso del que con mucho trabajo había podido sacar las copias y encuadernar" (S40).

Al consultarle al profesorado con relación a las posibilidades de continuar su carrera académica en Venezuela, las respuestas muestran que las perciben adversas, tal como se puede evidenciar en el gráfico 3.

Gráfico 3. Condiciones para desarrollar su carrera académica en la universidad

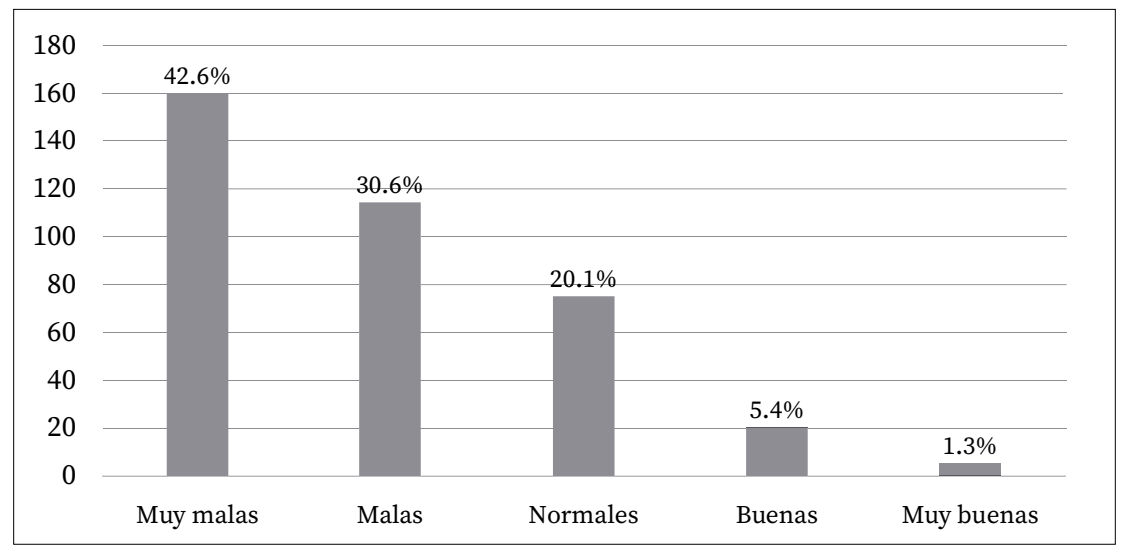

Fuente: Elaboración propia

El profesorado, mayoritariamente señala que las condiciones para estudiar o desarrollar su carrera académica eran, para el momento de su salida, malas o muy malas. Los que indicaron que 
eran muy malas especificaron: "No podía sentarme a leer o estudiar una nueva fórmula" (S05); "Las necesidades básicas eran apremiantes e impedían pensar en otra cosa" (S13); "Imposible la actualización profesional, ningún apoyo institucional y a nivel individual o mal comes o trabajas. Estudiar imposible por los bajos ingresos que tiene el profesional docente" (S16).

La idea de "malas" se corresponde con: "Imposible pensar en dedicarme exclusivamente a desarrollar mi carrera académica, ya entonces los sueldos eran miserables" (S28); "En la Universidad los medios no son adecuados, pero aun así, el arduo trabajo de algunos profesores permite que muchas carreras sigan a flote. Con mucho esfuerzo y de manera estrictamente individual se puede crecer en el campo académico venezolano, pero las condiciones del entorno entorpecen la lucha diaria para lograr las metas propuestas a corto y a largo plazo" (S41). La crisis institucional, la inexistencia de incentivos y la pauperización del salario afectaron la posibilidad de que los profesores continuaran su carrera académica: "Llegaba a dar clases después de caminar kilómetros, los pagos realizados por la UCV no compensaban ni un desayuno, tenía que hacer guardias nocturnas todas las noches y luego sin descansar ir a la escuela ... en estas condiciones no se puede ni vivir, mucho menos investigar y motivar a los estudiantes" (S347); “Todavía había oportunidades para progresar en la carrera académica dentro de la universidad, pero menos para cursar estudios en otras universidades y mucho menos en el exterior" (S302).

El 75,6\% de los participantes indicaron que en los dos últimos años de estancia en Venezuela se vio mermada su productividad académica. El gráfico 4 reporta el número de artículos que en promedio publicaban al año.

En este grupo de docentes, un poco más del $50 \%$ indica que publicaba en promedio, entre uno y dos artículos al año. Si computamos la categoría 9 o más, como 9 publicaciones al año, se tendría un total de 564 publicaciones y un promedio de 1,5 publicaciones por docente por año. La producción de artículos científicos por parte de investigadores venezolanos ha mantenido una tendencia a la baja desde 2009 (De la Vega y Vargas, 2014; Requena y Caputo, 2016; 
Ramírez y Salcedo, 2016) la cual ha sido ratificada en el Ranking iberoamericano de instituciones de educación superior 2020 (SIR Iber). Allí se señala que Venezuela muestra un crecimiento negativo que se expresa en "una disminución en el número de publicaciones, cercana a los 600 trabajos en el período 2014-2018, con relación al quinquenio 2013-2017” (De Moya-Anegón et al., 2020, p. 11).

Gráfico 4. Artículos publicados al año cuando estaban en Venezuela

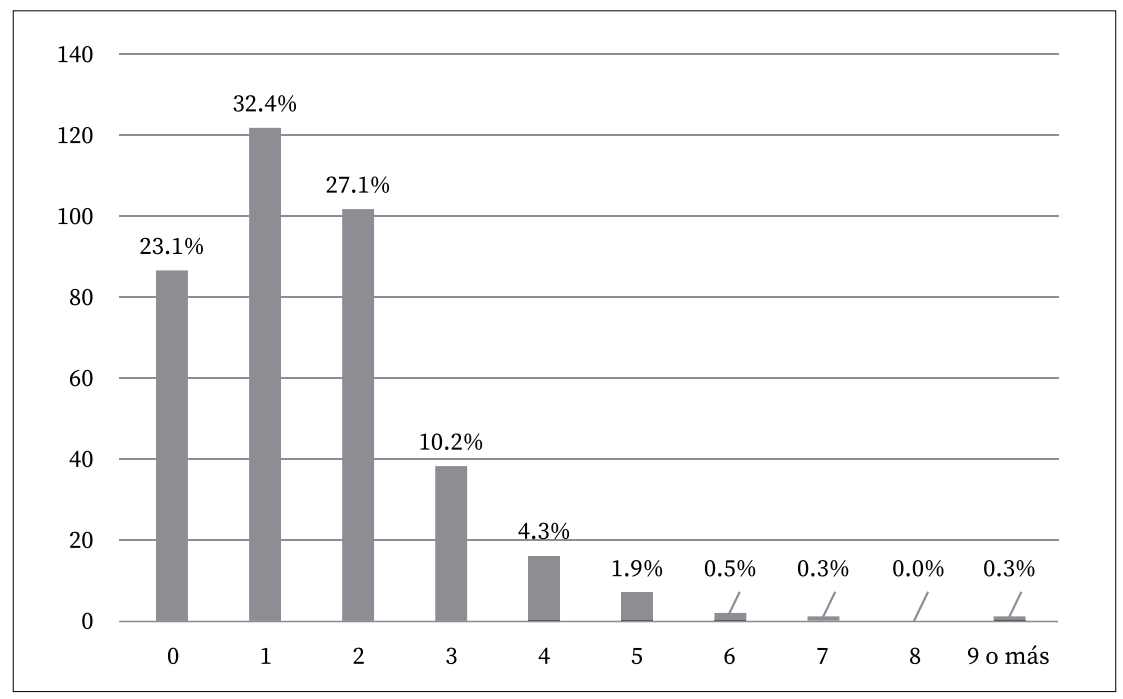

Fuente: Elaboración propia

Al consultarse las razones por las cuales lo profesores migrantes creen que durante los últimos años se vio afectada su productividad académica en Venezuela, las razones fueron variadas. Sobre estas razones se obtuvieron 100 respuestas. Entre ellas podemos destacar las que van desde la conflictividad política y social del país, la crisis humanitaria y la crisis universitaria. En términos de la conflictividad política, uno de los encuestados manifestó: "Situación irregular en el país, colas para la comida, transporte, escasez de recursos en la universidad e inasistencia de los profesores" (S55); "No había apoyo a la investigación (en cuanto a medios, infraestructura y tiempo)" (S07); 
"El hecho de tener que buscar trabajos alternativos para compensar el bajo salario" (S26); "Preocupaciones socioeconómicas, de salud y de tranquilidad ciudadana" (S181). También: "Situación del país, imposición del socialismo del siglo XXI -cambios sociopolíticos y económicos que resultaron en la precarización generalizada $\mathrm{y}$ creciente del ambiente de trabajo de la universidad y de la calidad de vida. Cambios e imposición de normativas que cercenaban la libertad académica, presupuestos reconducidos. Inseguridad, fallas en los servicios básicos/cortes de electricidad y de agua que impedían la permanencia en el instituto, imposibilidad de renovar equipos, asistir a congresos y pagar la afiliación a instituciones internacionales, entre otros" (S241).

Un aspecto importante señalado en los cuestionarios sobre la merma en la productividad académica del profesorado es la crisis humanitaria. Señala un encuestado: "Exigencias de vida extraacadémica inusuales (búsqueda de alimentos, múltiples problemas sociales y económicos), disminución de incentivos motivacionales y económicos en la universidad, caída de las condiciones mínimas de espacio e instrumentos institucionales para ofrecer clases adecuadamente" (S366). Así, la productividad académica es una variable importante dentro de la vida del profesor universitario, es un indicador que expresa su capacidad de generar conocimientos, tanto para la docencia universitaria, como para el avance y desarrollo de la ciencia venezolana en sus distintas disciplinas.

\subsection{Gestión Universitaria}

No solo la docencia y la investigación sufren con la salida de docentes de las instituciones universitarias. También se ve afectada la gestión universitaria y otras funciones necesarias para el normal desarrollo de los procesos académicos y administrativos propios de las instituciones. De los encuestados, $131(35,1 \%)$ señalaron que tenían funciones de gestión universitaria al momento de su salida del país. Las posiciones que ocupaban dentro de la estructura de gobierno universitario se resumen en la tabla 3. 
Tabla 3. Cargos que ocupaban en la gestión universitaria

\begin{tabular}{ll}
\hline Cargos & \\
\hline Consejo Universitario & 2 \\
Consejo de Facultad & 2 \\
Decano & 3 \\
Director & 23 \\
Coordinación & 69 \\
Jefatura de Departamento & 7 \\
Jefatura de Cátedra & 8 \\
Otros & 17 \\
\hline & 131 \\
\hline
\end{tabular}

Fuente: Elaboración propia

Buena parte de los emigrados ocupaban cargos intermedios en la gestión universitaria, vinculados principalmente a labores de coordinación. También han emigrado profesores que en su momento ocupaban cargos de coordinación académica, coordinación de postgrado, coordinación de maestría, coordinación de doctorado, coordinación de investigación, coordinación de servicio comunitario, coordinación de pasantías, coordinación de especialidad o de mención, coordinación de diseño curricular e instruccional. Hay también directores de escuela, de instituto, de centro o instituto de investigación, de escuela de postgrado, de formación y desarrollo, de cultura y de desarrollo estudiantil. Profesores en cargos de jefatura de departamento y de cátedra suman 15 encuestados. En el renglón "otros", hay profesores que desarrollaban funciones de apoyo directo a las labores universitarias como asesores, asistentes, representantes profesorales y secretarías académicas.

\subsection{La productividad académica en el país de acogida}

No todos los docentes emigrados se mantienen en actividades académicas en los países de acogida. Más de la mitad de los consultados 
señalaron que no laboran actualmente como profesor universitario. A las condiciones de vida del profesor, ahora migrante, que le condujeron a replantearse su proyecto de vida personal y profesional, se suman las nuevas condiciones por ser migrante: inseguridad jurídica, precariedad laboral, inestabilidad socioemocional. Es por ello que se les consultó cuántos trabajaban como profesor universitario y 147 de los 373 encuestados respondieron afirmativamente.

Ahora, ¿a qué se dedican los profesores venezolanos al migrar a otros destinos? En las respuestas dadas se pueden leer diversidad de ocupaciones que realizan para conseguir los recursos necesarios para sí y su grupo familiar. Algunos indicaron que teletrabajan administrando productos y servicios en la web, "trabajos en línea" (S37); muchos como "Consultora de investigación freelance" (S75); "Consultor de modelos de riesgos para una firma internacional” (S85); "Traductor" (S81). Otros profesores están empleados en labores de asistencia: "Asistente administrativo" (S182); "Asistente de clases" (S137); "Guardia de seguridad y asistente de productor cinematográfico y de espectáculos” (S251).

Otros han incursionado en servicios, ventas y oficios. Destacan afirmaciones como "cualquiera que la vida me ponga por delante" (S268); "No tengo trabajo fijo. Me he dedicado a labores agrícolas, a trabajos como ayudante o auxiliar de cocina" (S281); "Mixto, trabajo como profesora universitaria a tiempo parcial y lo combino con otro trabajo a tiempo parcial de mesera" (S333). En este grupo, podemos señalar algunas ocupaciones como: "Administradora de una empresa de eventos" (S237); "Amazon y Uber" (S121); "Anfitriona-cajera en KFC" (S267); "Vendedor de autos" (S32); "Bombero en una estación de gasolina” (S174). También en remodelación de inmuebles, obrero de construcción, cuidador de niños y adultos mayores, operador de servicios en empresa de lavado de automóviles.

Una parte de los migrantes labora en condición de dependientes o como autónomos y en oficios. Pocos indican estar desarrollando algún proyecto emprendedor. Destacan algunas referencias: "Inicié un emprendimiento" (S13); "Dueño de una pequeña tienda de ropa" (S179); "Gestiono un pequeño piso de mi propiedad, como piso turístico" (S331); "Tenemos una empresa consultora, mentores 
de emprendedores, capacitación laboral y reclutamiento de capital humano" (S34).

Otro segmento se desempeña en campos profesionales asociados a su formación con funciones de consultor, asesor o ejercicio libre de la profesión. Aquí los más frecuentes son los graduados en ingeniería o medicina. Ejemplo de las respuestas de este grupo son: "Consultor en Ernst \& Young" (S246); "Consultor internacional (GLG Group, Nueva York)” (S253); “Consultor educativo, diseñadora instruccional y tallerista" (S03); "Consultor/ asesor de sistema de gestión integrado" (S18). Hay casos de docentes que aún están desempleados, subempleados o en situación de dependencia, ya sea de pensiones familiares o pensiones de los sistemas de previsión social del país de acogida: "Actualmente estoy desempleada, dependo de mi esposo" (C326); "De manera muy sincera, no trabajo" (S352); "Ninguno que me proporcione una remuneración económica" (S14).

En el campo de la docencia directa, algunos profesores señalaron que en el país de acogida, se desempeñan en otros niveles de la educación, por ejemplo: "Maestro de escuela" (S337), "Maestro integrador -docente de educación especial” (S41); "Profesor de bachillerato" (S78); "Profesor y técnico de laboratorio en un liceo" (S131); "Profesora de high school" (S138).

Independientemente de si regresó o no a labores académicas en universidades, la mayoría $(84,7 \%)$ señala que su situación de vida es mejor o mucho mejor a la que tenía para el momento de su salida de Venezuela. Aún con las dificultades que significa ajustarse a un nuevo país, estos migrantes consideran que sus condiciones de vida actuales son superiores.

Al grupo que ya trabaja en universidades se le consultó sobre los factores que podrían haber facilitado su ingreso en esas instituciones. Sus respuestas se resumen en la tabla 4.

Aunque el principal factor señalado es la acreditación o formación académica, cuando se selecciona al personal docente de una universidad, no se examina únicamente su acreditación o formación académica, sino que se consideran otros elementos que conforman la carrera académica. Esto puede verse en algunas de las respuestas 
del grupo: "Una combinación compleja de todos los aspectos listados: alto nivel de formación y de experiencia científico-académica y redes profesionales a nivel internacional” (S29); "Tenía contactos con el rector y un ex rector, quienes decidieron apoyarme en mi salida del país" (S283); Ya tenía el contacto con la institución contratante desde Venezuela" (S194).

Tabla 4. Ingreso al campo de labor universitaria

\begin{tabular}{lcc}
\hline Factor & $\mathbf{N}$ & $\%$ \\
\hline Acreditación / Formación académica & 76 & 51,7 \\
Alta especialización & 22 & 15,0 \\
Experiencia & 14 & 9,5 \\
Vínculo o redes profesionales & 18 & 12,2 \\
Programa especial & 4 & 2,7 \\
Otros & 13 & 8,8 \\
\hline & & 147 \\
\hline
\end{tabular}

Fuente: Elaboración propia

Parte de los docentes que lograron insertarse en las universidades del país de acogida reportan que han tenido la posibilidad de continuar o iniciar investigaciones y dar evidencia de ese trabajo mediante publicaciones. Al consultárseles sobre las posibilidades de publicar artículos de investigación en sus condiciones actuales, señalan que las posibilidades son altas. En el contexto universitario de acogida, "más que posibilidades es mandatorio para trabajar en la universidad" (S75). Algunos, apenas iniciándose en las actividades universitarias señalan que "apenas acabo de ingresar a una universidad, tiempo parcial, no he podido desarrollar actividades de investigación, pero las condiciones son óptimas" (S342); "aun cuando no hay exceso de recursos y estos deben administrarse con mesura, siempre tengo recursos disponibles para mis requerimientos académicos" (S132). Algunos señalan que su dificultad mayor es epistemológica: "hacer un cambio del campo de problemas y migrar 
de la antropología cultural a las ciencias de la educación” (S83). Para algunos profesores es condición para la contratación tener publicaciones, particularmente en revistas de corriente principal (WOS, SCOPUS): "para renovarte el contrato es condición indispensable que tengas producción en investigación" (S360). Por lo tanto, se observa apoyo institucional pero además está la exigencia en materia de productividad científica.

El gráfico 5 muestra la comparación del promedio de artículos publicados al año, antes y después de salir de Venezuela, por los docentes que trabajan en universidades.

Gráfico 5. Artículos publicados al año antes y después de salir de Venezuela

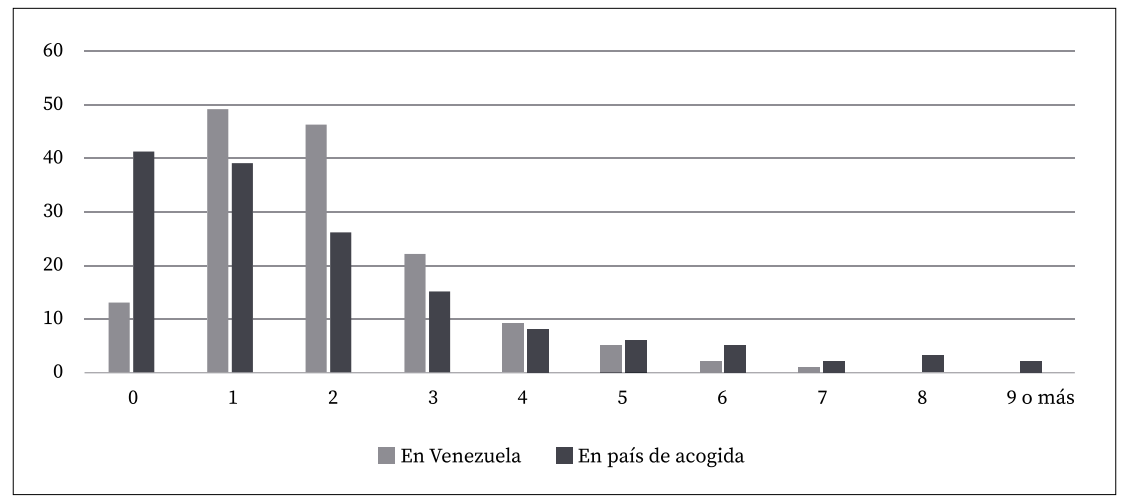

Fuente: Elaboración propia

El gráfico anterior se puede ver en dos partes. En los valores bajos de la variable, 1, 2, 3 y 4 artículos al año, la producción era mayor en Venezuela. Mientras que para 5 o más artículos al año, la producción es superior en el país de acogida. También, el número de docentes que no publica es mayor en el país receptor. Este grupo de 147 docentes, según su testimonio, en Venezuela publicaba 287 artículos por año, mientras en el país de acogida ha publicado 284 artículos por año, eso significa una disminución de 1,05\% de su producción escrita. Una variable implícita en esta situación es el tiempo de estadía que tiene el profesor en el país receptor, desempeñándose 
como profesor universitario. Es así como de los 41 docentes que declara no tener artículos publicados, más de la mitad, 27, tiene un año o menos fuera de Venezuela. Mientras que aquellos que reportan haber publicado 5 o más artículos, en su mayoría tienen 3 o más años en el nuevo país de residencia.

Los encuestados, que actualmente están trabajando en universidades, señalan que las instituciones están muy atentas a fomentar la investigación e igualmente son exigentes con su profesorado en materia de publicación: "eso es considerado en nuestra evaluación semestral y, por ende, te mantienen motorizados todos los procesos que conducen a su logro contando incluso, con apoyo al diseño y a las publicaciones de revistas y de textos" (S207). Los sistemas de producción, gestión y divulgación de conocimiento son exigentes: "En Brasil las revistas se clasifican en A, B y C, siendo que las A son las de mayor credibilidad y calidad, y así van decreciendo en valor y grado. Se puede publicar en inglés, portugués o español, según la revista; en el contexto académico en que me encuentro se requiere de la publicación de al menos tres artículos de investigación al año" (S219).

Las respuestas indican que ha mejorado la productividad académica de aquellos que ingresaron a las universidades y tienen mayor tiempo en ellas. Ellos señalan que ahora tienen incentivos para investigar y publicar, cuentan con apoyo financiero para asistir a eventos académicos, lo que favorece la investigación. Consideran que las universidades donde trabajan apoyan la investigación y la publicación de artículos: "Hay un interés especial por favorecer las publicaciones tanto del personal docente como de los estudiantes de maestría" (S111). "Es política de la universidad que todos sus docentes realicen investigación; por esa razón un tercio de las horas de trabajo se dedican a la investigación" (S24). También hay "facilidad de acceso a bases de datos internacionales y de trabajar en proyectos de investigación” (S.121). Existen grupos de innovación e investigación docente, estimulados y apoyados económicamente desde las universidades. Es decir, "todo el sistema está 'montado' para que se produzca investigación” (S74). Entonces, pese al cambio que significa emigrar, algunos profesores universitarios han logrado dar continuidad a la carrera académica iniciada en Venezuela. 


\section{Conclusiones}

Según organismos internacionales, como consecuencia de la severa crisis política, social, económica e institucional, en los últimos años han emigrado de Venezuela más de 5 millones de ciudadanos. A diferencia de lo que ocurría en 1999, donde emigraban empresarios y ciudadanos de la clase media mayormente, ahora la salida de venezolanos abarca por igual a todos los sectores y estratos sociales. Un sector que ha aumentado su salida en los últimos años es el de docentes de todos los niveles; en este artículo hemos observado a los del sector universitario. Las limitaciones que impone la muestra no probabilística obligan a ser cautelosos con las conclusiones.

Distintos elementos conforman la carrera académica del docente: el área de conocimiento donde investiga, el grado académico alcanzado, la categoría profesional, antigüedad y experiencia en investigación, antigüedad en su universidad (Aceytuno y SánchezLópez, 2014), entre otros. Pero también influyen los circuitos donde visibilizan sus investigaciones, la asistencia a eventos y su inserción en redes académicas nacionales e internacionales. Todos estos son factores que ayudan al profesorado a estructurar su carrera académica, la cual tiene influencia en su productividad y en las posibilidades de implicarse en actividades de transferencia de tecnología. De acuerdo con los migrantes consultados, en Venezuela había dificultades de llevar adelante una carrera académica y continuar su productividad en investigación.

Habiéndose dedicado a la vida académica, a la producción de investigación y a la gestión universitaria, muchos decidieron salir del país, ¿por qué emigraron? Lo hacen por varias razones, como la búsqueda de un mejor futuro para ellos y sus familias o recobrar un nivel de vida decorosa, pero en la base está la idea de continuar con su vida académica. La formación lograda y su opción por la docencia universitaria los lleva a buscar oportunidades que permitan la continuación de su carrera en mejores condiciones. En este sentido, la información lograda en esta muestra parece coincidir con la idea planteada por Mesa y Phelan (2018) en cuanto a que el profesional venezolano emigra porque quieren dar valor a las capacidades adquiridas. 
Los profesores universitarios de esta muestra se esfuerzan por insertarse en los sistemas universitarios de los países receptores. En la medida en que lo hacen, ponen su disposición y talento para recuperar la producción científica que habían perdido en Venezuela. No todos logran ingresar a las universidades, por lo que algunos se ven obligados a desempeñar diversas actividades en las sociedades receptoras. Esas experiencias fortalecen sus capacidades personales. La migración calificada puede sufrir de limitaciones en los países receptores, sobre todo en el caso venezolano donde la migración de profesionales es masiva y no selectiva. El migrante debe abrirse paso en las sociedades de acogida para que su experiencia y credenciales sean reconocidas. Para los países receptores, aunque la migración calificada pueda ser bien vista, al no ser planificada y masiva puede ser un problema para el país receptor.

Por otra parte, la investigación y en consecuencia la publicación de artículos científicos es otra función de la universidad venezolana que posiblemente se resienta con la salida de los profesores, la mayoría de ellos con maestría o doctorado y publicaciones regulares. Este fenómeno tiene dos consecuencias, una directa; la pérdida de investigaciones y publicaciones que producía ese profesorado, y una indirecta; la formación de nuevos investigadores. El camino a la investigación se allana cuando se trabaja "junto a un maestro/a, como en los gremios medievales, dentro de un proyecto de trabajo dirigido por el maestro/a. Es así porque hay algo no codificable del oficio del investigador, difícil de transmitir si no es en el hacer" (Wainerman y Sautu, 2011, p. 30).

Las respuestas de los profesores migrantes que se han incorporado a universidades en los países receptores indican que han podido recomponer sus carreras académicas, lograr apoyo a la investigación y continuar aportando a la producción de conocimientos. Pareciera entonces que la migración es una oportunidad para recuperar la productividad mermada o perdida ante el cambio en las condiciones de vida personal y profesional.

Se podría esperar que el esfuerzo personal de los migrantes, en el mediano o largo plazo, redunde en el desarrollo de la ciencia venezolana. Esto según se desprende del planteamiento en el cual 
la migración calificada, si bien puede ser considerada una pérdida de capacidades para la sociedad de origen, si es canalizada adecuadamente, con políticas públicas e institucionales de retorno o de trabajo en red, puede ser una oportunidad para el ecosistema científico tecnológico del país receptor y del emisor de migrantes. Para ello es necesario que en Venezuela cambien las condiciones que provocaron la emigración de académicos, científicos y de la población en general.

\section{Referencias}

Aceytuno, M. y Sánchez-López, C. (2014). Carrera académica y propensión a la transferencia de tecnología: el caso de la Universidad de Huelva. Investigaciones Regionales, 28, 79-100.

ACNUR (2020). Situación Venezuela. Consultado en julio de 2020. Disponible en: https://www.acnur.org/situacion-en-venezuela.html

Aula Abierta (2018). Informe: Decisiones del poder judicial que atentan contra el principio de autonomía universitaria y la libertad académica en Venezuela. Aula Abierta. Disponible en: https://bit.ly/2NggBPl.

Bravo, L. (2019). Educación Universitaria en Venezuela 2018. Disponible en: https://app.box.com/s/x3vtssfug12mimn15jth4qn4sd48yp7n/ file/503128684872.

Bravo, L. (2015). Educación Universitaria en Venezuela 2014. Disponible en: https://app.box.com/s/x3vtssfug12mimnl5jth4qn4sd48yp7n/ file/503115129875.

Castro, J. [@juliocastrom] (2020). Robo \#76 en el Instituto de Medicina Tropical. [Tweet]. Twitter. https://twitter.com/juliocastrom/status/1247163025204731905

Cohen, J. (2001). Transnational migration in rural Oaxaca, Mexico: dependency, development and the household. American Anthropologist, 103, 954-967.

Consultores 21 (2019). Diáspora. Servicio de análisis de entorno $\mathrm{N}^{\circ} 7$. Cobertura Urbano Rural. : http://venamerica.org/web/wp-content/ uploads/2019/09/CONSULTORES_21_Diaspora_2do_trimestre_2019.pdf 
Coordinadora Regional de Investigaciones Económicas y Sociales (CRIES) y Stanley Foundation. (2017). Policy Memo-Respuestas Regionales a la Crisis en Venezuela. Disponible en: http://www.cries.org/wp-content/ uploads/2017/08/Policy-Memo_Venezuela-Crisis_Spanish-1.pdf

De la Vega, I. y Vargas, C. (2014). Emigración intelectual y general en Venezuela: una mirada desde dos fuentes de información. Bitácora-e revista electrónica latinoamericana de estudios sociales, históricos y culturales de la ciencia y la tecnología, 1, 66-92.

De Moya-Anegón, F., Herrán-Páez, E., Bustos-González, A., Corera-Álvarez, E., Tibaná-Herrera, G. y Rivadeneyra, F. (2020). Ranking iberoamericano de instituciones de educación superior 2020. Granada: Ediciones Profesionales de la Información.

Domeniconi, J, y Jarochinski Silva, J. C. (2018). A imigracao quialificada venezuelana do seculo XXI: um estudo a partir dos espacos da migracao de trabalhadores do conhecimiento da Venezuela no Brasil. En R. Baeninger y J.C. Jarochinsky (Eds.) Migracoes Venezuelanas (pp.333-346). Universidade Estadual de Campinas.

Existe un $40 \%$ de deserción de profesores y alumnos en las Universidades (08 de marzo de 2020). Descifrado. Recuperado de: http://www.descifrado.com/2020/03/08/existe-un-40-de-desercion-de-profesores-y-alumnos-en-las-universidades/

Freitez, A. (2019). Venezuela. Transición de un proceso de emigración calificada a migración masiva. En Observatorio Venezolano de Migración/ Instituto de Investigaciones Económicas y Sociales (Coodinadores). Horizontes de la emigración venezolana: Retos para su inserción laboral en América Latina. Universidad Católica Andrés Bello, Caracas, Venezuela.

García, M. y Restrepo, J. (2019). Aproximación al proceso migratorio venezolano en el siglo XXI. Hallazgos. 16, 63-82.

Hernández R., Fernández, C., y Baptista, P. (2006). Metodología de la investigación. México: McGraw Hill.

La UCV tendrá presupuesto reconducido por $7^{\circ}$ año (Octubre 2013). Hora Universitaria, Recuperado de: http://www.ucv.ve/fileadmin/user_upload/ dic/Publicaciones/Hora_Universitaria/HORAUnive._OCTUB2013.pdf

Lares, C. (2014). Estudiantes venezolanos de maestría y doctorado en el extranjero: intención de regresar a Venezuela y contribuir a la construcción del país que desean. En: Peralta, R., Lares, C. y Vegas, K. (Eds.). Diás- 
pora del talento. Migración y educación en Venezuela: análisis y propuestas, (pp. 111-150). Venezuela-FUNDACELAC.

León, J. (2014). Dificultades para la implementación de la Ley Orgánica de Ciencia, Tecnología e Innovación (LOCTI) en la Universidad Central de Venezuela. Revista de la Facultad de Ingeniería. 29, 7-11.

Linarez, G. y Linarez, G. (2019). Éxodo del docente universitario en Venezuela. Revista Scientific. 4, 141-162.

Masanet, E. y Moncusí-Ferré, A. (2020). La migración cualificada en dirección Norte-Sur: el caso de los científicos y científicas de origen español en Ecuador durante la Gran Recesión (2008-2015). Disjuntiva. 1, 49-65.

Mesa, C. y Phelan, M. (2018). El fenómeno de la emigración internacional en Venezuela. Una mirada desde el enfoque de las capacidades y de desarrollo humano. Analogías del comportamiento, 16, 28-43.

Ministerio de Ciencia y Tecnología (2005). Plan Nacional de Ciencia, Tecnología e Innovación 2005-2030. Recuperado de: http://biblioteca.vtv.gob. ve/greenstone/collect/historica/index/assoc/HASH3aec.dir/doc.pdf

Morles, V., Medina., E. y Álvarez., N. (2003). La educación superior en Venezuela: Informe 2020. Recuperado de: https:/unesdoc.unesco.org/ ark:/48223/pf0000131594

Oficina del Alto Comisionado de las Naciones Unidas para los Refugiados (2020). Situación Venezuela. Recuperado de: https://www.acnur.org/situacion-en-venezuela.html

Organización Internacional para las Migraciones (2018). Plan de Acción Regional. Recuperado de: https://www.refworld.org.es/pdfid/5bb53ef84.pdf

Organización Internacional para las Migraciones (2012). Gestión Fronteriza Integral en la Subregión Andina. Módulo de capacitación para una gestión fronteriza integral que garantice los derechos humanos de las personas en movilidad y combata la trata de personas y el tráfico ilícito de migrantes. Organización Internacional para las Migraciones y Comisión Andina de Juristas. Lima, Perú: PER-OIM. Recuperado de: https:// repository.oim.org.co/handle/20.500.11788/1504

Organización de las Naciones Unidas para la Educación, la Ciencia y la Cultura (UNESCO) (2019) Migración, desplazamiento y educación: construyendo puentes, no muros. Informe de seguimiento de la educación en el mundo. Recuperado de: https://unesdoc.unesco.org/ark:/48223/ pf0000367436 
Páez, T. y Phelan, M. (2019). Emigración venezolana hacia España en tiempos de Revolución Bolivariana (1998-2017). RIEM, Revista Internacional de Estudios Migratorios, 8, 319-355.

Páez, T. (27 al 29 de mayo de 2014). La diáspora venezolana: políticas públicas y desarrollo. Asociación Venezolana de Gestión e Investigación y Desarrollo y Asociación Internacional de Gestión de Investigación y Desarrollo (coordinadores). VII Reunión Internacional de Gestión de Investigación y Desarrollo, Universidad de Granada, Facultad de Comunicación y Documentación, Campus Universitario de Cartujas, Granada, España.

Parra, M. (2003). La profesión académica en Venezuela: de los «catedráticos» a los profesores universitarios. Cuadernos del Cendes. 20, 93-120

Pellegrino, A. Bengochea, J. y Koolhaas, M. (2013). La migración calificada desde América latina. Tendencias y consecuencias. Montevideo: Programa de Población Unidad Multidisciplinaria. Facultad de Ciencias Sociales-Universidad de la República. Uruguay.

Ramírez, T. (2020). La universidad autónoma venezolana y su lucha por sobrevivir al socialismo del siglo XXI. Cinco propuestas para su rescate. Universidades, 71, 53-72.

Ramírez, T. y Salcedo, A. (2016). Inversión y producción científica en Venezuela ¿una relación inversamente proporcional? Revista de Pedagogía, 37, 147-174.

Requena, J. y Caputo, C. (2016). Pérdida de talento en Venezuela. Migración de sus investigadores. Interciencia, 41, 444-453.

Rondón, N. (03 de noviembre de 2017). Encapuchados asaltaron a estudiantes de la UDO. El Mercurio Web. Recuperado de: https://elmercurioweb.com/noticias/2017/11/3/encapuchados-asaltaron-a-estudiantes-de-la-udo.

Sánchez, M. y Massey, D. (2014). Migración de talento y profesionales cualificados: el caso reciente de inmigrantes venezolanos a EE.UU. En Peralta, R., Lares, C. y Vegas, K. (Eds.). Diáspora del talento, migración y educación en Venezuela: análisis y propuestas (pp. 31-56). Venezuela: FUNDACELAC.

Singer, F. y Moleiro, A. (27 de junio 2020). La lucha por salvar las universidades de Venezuela del abandono. El País. Recuperado de: https://elpais. com/cultura/2020-06-27/la-lucha-por-salvar-las-universidades-de-venezuela-del-abandono.html 
Torres, L., Stephany, K. y Parra-Sandoval, M. (2020). El gobierno venezolano y la política salarial implementada a los profesores universitarios (20002018). Universidades, 71, 73-91.

Tovar, F. (16 de agosto de 2020). Profesores de la Universidad del Zulia están pasando hambre. Portal de Noticias Costa del Sol. Recuperado de: https:// www.costadelsolfm.org/2020/08/16/profesores-de-la-universidad-del-zulia-estan-pasando-hambre/

Universidad Simón Bolívar. (09 de marzo de 2020). USB en Breve. Recuperado de: http://www.usb.ve/home/node/6168

Vargas, C. (2018). La migración en Venezuela como dimensión de la crisis. Pensamiento Propio, 47, 91-130.

Wainerman, C. y Sautu, R. (2011). La trastienda de la investigación. Buenos Aires: Manantial.

Zuñiga, G. (septiembre 2011). La emigración de jóvenes en Venezuela: problemas y soluciones. Friedrich Ebert Stiftung en Venezuela (coordinador). La Nueva Agenda de la Juventud Social demócrata en Venezuela: Retos y Desafíos. Caracas, Venezuela.

Recibido: 30.06.2020 Aceptado: 24.09.2020 